\title{
SIGNAL PROCESSING DEVELOPMENTS FOR THE OVRO ARRAY
}

\author{
STEPHEN PADIN and SCHUBERT F. SOARES \\ California Institute of Technology, Owens Valley Radio Observatory, Big Pine, \\ California. 93513. USA.
}

\begin{abstract}
An optical fiber IF transmission and tracking delay system and a wideband continuum correlator have been developed for the Owens Valley (OVRO) Millimeter Array. The IF system processes two 1-2 GHz bands which are frequency multiplexed through an optical fiber link. This allows simultaneous dual wavelength observations in the 2.7 and $1.3-\mathrm{mm}$ bands or dual polarization observations in either band.
\end{abstract}

\section{INTRODUCTION}

A dual channel IF system has been developed for the OVRO Millimeter Array to allow dual polarization and dual wavelength observations. For dual polarization observations the receivers in the telescopes are configured with two identical SIS mixers which are fed with orthogonal linear polarizations by the telescope optics (Scoville 1992). The IF bands from the mixers are transmitted to correlators via an optical fiber link. The OVRO digital spectral line correlator can be configured for dual polarization observations with up to $256 \mathrm{MHz}$ bandwidth and 256 lags for each polarization. This is an important operating mode since it provides a $\sqrt{2}$ increase in the sensitivity of the instrument.

For dual wavelength operation the receivers are fitted with one $2.7-\mathrm{mm}$ mixer and one 1.3-mm mixer. Since the OVRO Array has only one local oscillator reference the two local oscillator frequencies must be related. Thus, dual wavelength continuum observations can be made but line observations are restricted to one wavelength.

The signal processing arrangements for two IF channels are shown in Fig. 1. The IF bands from the receivers in a telescope are frequency multiplexed through a single optical fibre link which contains the three longest segments of the tracking delay. The remaining delay segments are microwave circuits. Following the delay there is a demultiplexer which separates the two IF bands. Functionally the scheme is equivalent to two IF transmission systems and two tracking delays for each telescope in the array. The frequency multiplexing scheme is more complicated but is less expensive because it requires only one optical link. Also, the wide-band link and delay required for frequency multiplexing provides a route for increasing the bandwidth of the instrument in the future.

\section{OPTICAL FIBER IF LINKS}

Optical fiber links are attractive for IF transmission in an array because they have wide bandwidths and small passband errors. The optical fiber links in the OVRO Array are 


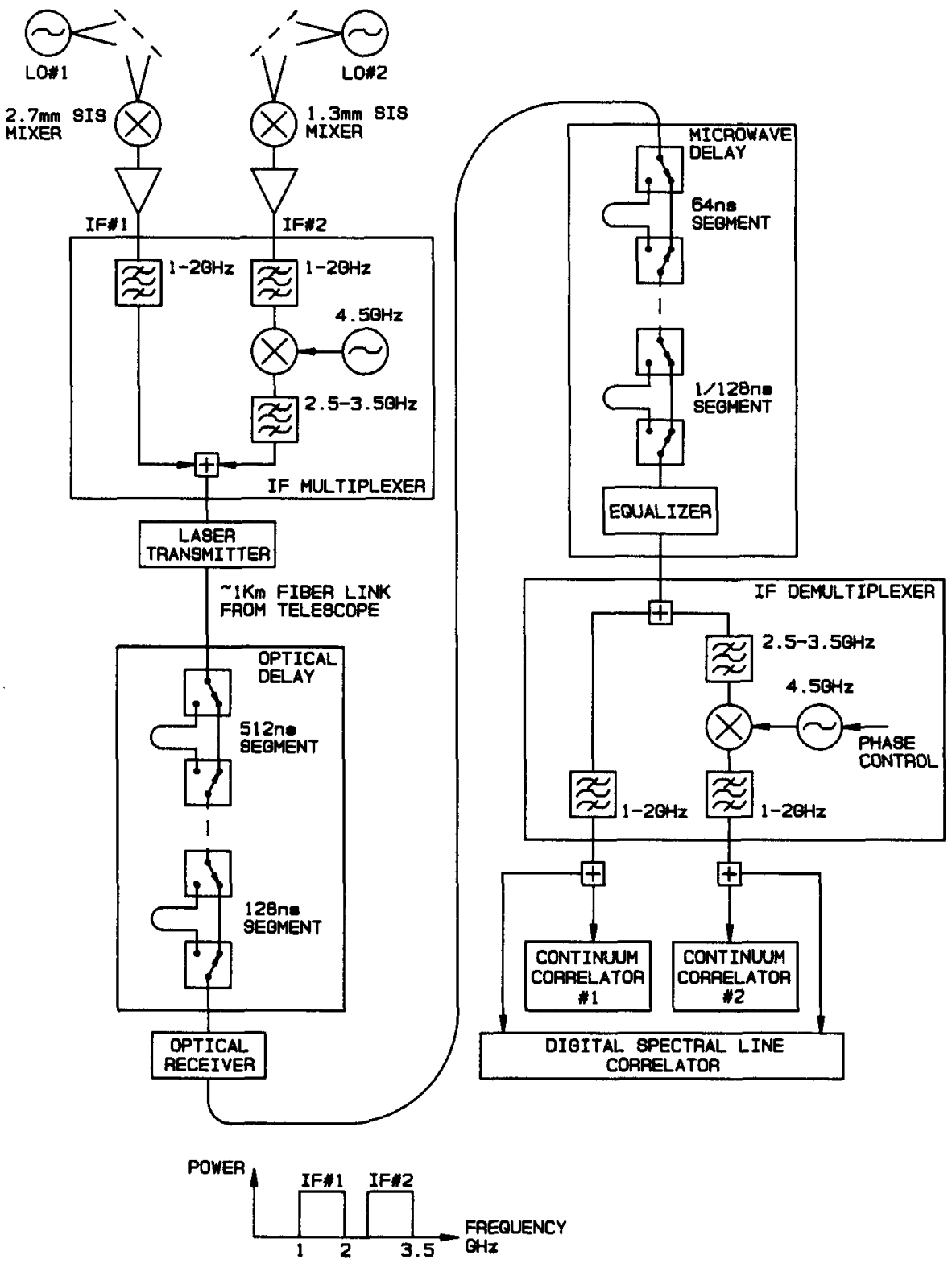

Fig. 1. IF system for one telescope in the OVRO Array. The configuration shown is for dual wavelength operation. For dual polarization observations two identical SIS mixers are used and these are fed with orthogonal polarizations. The IF processor in the digital spectral line correlator contains switches which assign correlator modules to one of the two IF channels. 
commercially available $1310-\mathrm{nm}$ singlemode units with $\sim 10 \mathrm{GHz}$ bandwidth. Singlemode fiber was chosen because it supports very large bandwidths. The OVRO Array is cabled with phase-stabilized optical fiber which has a liquid crystal polymer coating with a thermal expansion coefficient that compensates for that of the silica fiber (Sato 1992). The transmission delay variation with temperature is $\sim 50 \mathrm{ps} / \mathrm{Km}$ for a 10 to $40^{\circ} \mathrm{C}$ temperature change and $\sim 10 \mathrm{ps} / \mathrm{Km}$ for a -10 to $+10^{\circ} \mathrm{C}$ change. While most of the fiber in the array is underground there is a $100-\mathrm{m}$ exposed section for each telescope. In a 24-hour period the delay variation for the exposed section is $\sim 5 \mathrm{ps}$ in summer but only $\sim 1 \mathrm{ps}$ in winter. The differential effect between telescopes is smaller since the temperature variations for the exposed fibers are at least partially correlated. The worst case summer diurnal delay variation results in a phase change of $\sim 6^{\circ}$ at the correlator output for an IF frequency of 3.5 $\mathrm{GHz}$. The temperature induced delay variations are small enough to allow the fiber to be used for distributing the reference frequency for the millimeter-wave local oscillators: The winter delay variation of $1 \mathrm{ps}$ corresponds to a local oscillator phase variation of 0.23 turns at a wavelength of $1.3 \mathrm{~mm}$.

The useful bandwidth of an optical fiber link is determined by the link noise and the maximum input power for linear operation. For a lossy optical fiber link the link noise is dominated by shot noise in the photodetector while in low-loss links noise generated in the laser is important. The links in the OVRO Array have an equivalent input noise of -115 $\mathrm{dBm} / \mathrm{Hz}$ with an optical loss of $8 \mathrm{~dB}(2.5 \mathrm{~dB}$ for the fiber and connectors which link a telescope to the correlator building and $5.5 \mathrm{~dB}$ for the three longest segments of the tracking delay). The maximum drive level for the laser transmitter is $+13 \mathrm{dBm}$ but the normal operating point is $+3 \mathrm{dBm}$ which allows for power variations of $+10 \mathrm{~dB}$ due to the atmosphere, receiver gain variations and inserting a hot load for system noise temperature measurements. With a total of $+3 \mathrm{dBm}$ in the two $1-\mathrm{GHz}$ wide IF bands the power spectral density is $-90 \mathrm{dBm} / \mathrm{Hz}$ which is $25 \mathrm{~dB}$ above the noise floor. An IF power variation of -10 $\mathrm{dB}$ relative to the normal operating point degrades the system noise temperature by $3 \%$. The design details for the OVRO optical links show that it is difficult to achieve a high signal-tonoise ratio with a very wide-band IF link even for a modest IF dynamic range of $20 \mathrm{~dB}$. The required IF dynamic range could be reduced by continuously adjusting the link input power. In the OVRO system this would allow the full $10 \mathrm{GHz}$ link bandwidth to be used. However, controlling the link input power complicates the system because level control devices do not usually have constant transmission phase.

\section{TRACKING DELAYS}

The OVRO Array uses wide-band analog tracking delays primarily because these devices interface well to wide-band analog continuum correlators. Digital delays are much easier to manufacture but are relatively narrow-band so a scheme with multiplexing or many IF subbands is needed to process a large bandwidth.

The longest segments of an analog delay are generally the most difficult to realize because they introduce both the largest passband errors and the largest delay variations with temperature. In the OVRO delays these problems have been reduced by including the three longest delay segments $(512,256$ and $128 \mathrm{~ns})$ in the optical fiber IF links. Adding an extra length of fiber in a link has a negligible effect on the link passband errors and since the delay segments are realized in phase stable fiber the temperature problem is greatly reduced.

Unfortunately the mechanical fiber switches used in the OVRO delays are lossy ( 1 to $2 \mathrm{~dB}$ per segment) and this reduces the signal-to-noise ratio for the links and hence limits the number of optical delay segments. 
The shorter delay segments in the OVRO system are realized in coaxial and microstrip transmission lines (Soares and Padin 1992). The smallest delay step is $1 / 128 \mathrm{~ns}$ and the maximum delay error is $\sim 5 \mathrm{ps}$ which gives a visibility phase error of $6^{\circ}$ for signals at the top of the 2.5 to $3.5 \mathrm{GHz}$ IF band in Fig. 1. A high resolution delay was chosen to avoid having to compute the delay error and correct the amplitude and phase of the correlator outputs. Since the visibility phase error due to a tracking delay error is determined by the transmission frequency, the multiplexed IF scheme used in the OVRO Array increases the required delay precision. This increases the cost and difficulty of manufacture but the OVRO delay can be used in a 3-GHz bandwidth system in the future.

\section{WIDEBAND CONTINUUM CORRELATOR}

The OVRO Array currently has a 1-GHz bandwidth analog continuum correlator and a similar correlator is being constructed for the second IF band. For each baseline in the array there is a complex multiplier which consists of two microwave mixers, one fed in phase from a pair of telescopes and the other fed in quadrature (via a $90^{\circ}$ hybrid). The circuit was originally developed for a single-sideband mixer for the baseband downconverters in the OVRO digital correlator (Padin 1992). The multipliers are fed by level control circuits which are included to avoid errors due to changes in the multiplier operating point.

The multipliers have passband errors of $\sim 1 \mathrm{~dB}$ and $\sim 5^{\circ}$ across the 1 to $2 \mathrm{GHz}$ IF band, resulting in a sensitivity degradation of $\sim 2 \%$. The real and imaginary channels have relative amplitude errors of $\sim 3 \%$ and the quadrature error is typically $3{ }^{\circ}$. These errors are baseline-based and introduce small closure errors which must be calibrated. This is a disadvantage of complex correlators which use an analog hybrid to generate the quadrature channel. The advantages of the complex analog continuum correlator are its wide bandwidth, simplicity and relatively low cost.

\section{CONCLUSIONS}

A wide-band IF system using optical fiber links and fiber tracking delays has been developed for the OVRO Millimeter Array. The system processes two 1-GHz bandwidth IF channels to allow dual wavelength and dual polarization observations. The prototype system is currently installed on one telescope and the entire array will be upgraded in Spring 1993.

\section{ACKNOWLEDGEMENTS}

The OVRO Millimeter Array is partially supported by NSF grant AST 91-00306.

\section{REFERENCES}

Padin, S., Arend, B. and Narayanan, G. 1992, Microwave J., 35, 131.

Sato, K. et al. 1992, IEEE Trans. IM, IM-41, 385.

Scoville, N.Z. et al. 1992, ibid.

Soares, S.F. and Padin, S. 1992, ibid. 
Peter Hall: How often do you anticipate having to re-make the connectors on the monomode fiber?

Steve Padin: Each time a telescope is moved. This is $\sim 10$ times per year so a single-mode connector with a lifetime of a few hundred matings should last at least 10 years. 